Acta Crystallographica Section E

Structure Reports

Online

ISSN 1600-5368

\title{
4-Aminopyridinium isonicotinate monohydrate
}

\section{Alan R. Kennedy* and Madeleine Kittner}

Department of Pure and Applied Chemistry, University of Strathclyde, Glasgow G1 1XL, Scotland

Correspondence e-mail:

a.r.kennedy@strath.ac.uk

\section{Key indicators}

Single-crystal $\mathrm{X}$-ray study

$T=123 \mathrm{~K}$

Mean $\sigma(\mathrm{C}-\mathrm{C})=0.002 \AA$

$R$ factor $=0.036$

$w R$ factor $=0.090$

Data-to-parameter ratio $=11.3$

For details of how these key indicators were automatically derived from the article, see http://journals.iucr.org/e.
The 4-aminopyridinium isonicotinate salt was isolated as a monohydrate, $\mathrm{C}_{5} \mathrm{H}_{7} \mathrm{~N}_{2}^{+} \cdot \mathrm{C}_{6} \mathrm{H}_{4} \mathrm{NO}_{2}^{-} \cdot \mathrm{H}_{2} \mathrm{O}$, with a layered cation-water-anion structure. Hydrogen-bonding between layers utilizes all hydrogen-bonding donors and acceptors, whilst $\pi$ stacking dominates interactions within the organic layers.

\section{Comment}

The isonicotinate anion is well known as a ligand capable of forming supramolecular metal-organic structures. Here, the serendipitous isolation of the title compound, (I), highlights that it is also capable of supporting organic supramolecular architectures. Isolated with the 4-aminopyridinium cation [itself well known in studies of organic supramolecular structures; see for example Cowan et al. (2001) and Teulon et al. (1985)], (I) is found as a monohydrate (Fig. 1) with, in the $c$ direction, alternating layers of cations and anions separated by water molecules (Fig. 2). These layers are held together by the participation of all the molecular fragments in a threedimensional hydrogen-bonding network. Each cation acts as a threefold donor using all its $\mathrm{N}-\mathrm{H}$ bonds, each anion acts as a fourfold acceptor with atom $\mathrm{O} 1$ accepting two hydrogen bonds and atoms $\mathrm{O} 2$ and N1 accepting one each, and the water molecule both accepts a single hydrogen-bond and acts as a twofold donor. Within each organic layer, $\pi$ stacking interactions are observed. The shortest such contacts appear between antiparallel 4-aminopyridinium cations, with a centroid-to-centroid distance of $3.473 \AA$, whilst the isonicotinate anions are separated by $3.520 \AA$.<smiles>Nc1cc[nH+]cc1</smiles><smiles>O=C([O-])c1ccncc1</smiles>
$\mathrm{H}_{2} \mathrm{O}$

(I)
Received 5 January 2005 Accepted 10 January 2005 Online 22 January 2005

\section{Experimental}

Compound (I) was formed during an attempt to prepare $N$-(pyridine4-methylene)pyridin-4-amine. 4-Aminopyridine (1.24 g, $13.2 \mathrm{mmol})$ was dissolved in anhydrous xylene $(80 \mathrm{ml})$, and pyridine-4-carbaldehyde $(1.3 \mathrm{ml}, 13.6 \mathrm{mmol})$ and acetic acid $(0.38 \mathrm{ml}, 6.6 \mathrm{mmol})$ were added dropwise with stirring. The mixture was heated to reflux in a Dean-Stark apparatus for $20 \mathrm{~h}$. After removing the solvent in vacuo, a yellow oil was obtained. Crystalline (I) was obtained from a 


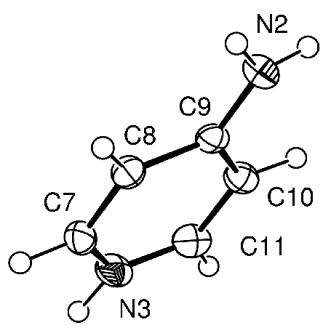

Figure 1

Asymmetric unit of (I), shown with $50 \%$ probability displacement ellipsoids.

chloroform solution of this oil after layering with diethyl ether (1.64 g, 53\% yield). IR (KBr, cm $\left.{ }^{-1}\right): 677,769,1204,1373,1542,1603$, 1650,3415 .

\section{Crystal data}

$\mathrm{C}_{5} \mathrm{H}_{7} \mathrm{~N}_{2}^{+} \cdot \mathrm{C}_{6} \mathrm{H}_{4} \mathrm{NO}_{2}^{-} \cdot \mathrm{H}_{2} \mathrm{O}$
$M_{r}=235.24$
Triclinic, $P \overline{1}$
$a=6.7128(3) \AA$
$b=6.7911(2) \AA$
$c=13.6379(8) \AA$
$\alpha=75.830(3)^{\circ}$
$\beta=75.999(2)^{\circ}$
$\gamma=78.326(3)^{\circ}$
$V=578.08(5) \AA^{\circ}$

$$
\begin{aligned}
& Z=2 \\
& D_{x}=1.351 \mathrm{Mg} \mathrm{m}^{-3} \\
& \text { Mo } K \alpha \text { radiation } \\
& \text { Cell parameters from } 2294 \\
& \quad \text { reflections } \\
& \theta=1.0-26.4^{\circ} \\
& \mu=0.10 \mathrm{~mm}^{-1} \\
& T=123(2) \mathrm{K} \\
& \text { Cut plate, colourless } \\
& 0.45 \times 0.37 \times 0.10 \mathrm{~mm}
\end{aligned}
$$

\section{Data collection}

Nonius KappaCCD diffractometer $\omega$ and $\varphi$ scans

Absorption correction: none

9253 measured reflections

2348 independent reflections

1848 reflections with $I>2 \sigma(I)$

\section{Refinement}

Refinement on $F^{2}$

$R\left[F^{2}>2 \sigma\left(F^{2}\right)\right]=0.036$

$w R\left(F^{2}\right)=0.090$

$S=1.04$

2348 reflections

207 parameters

All H-atom parameters refined

$$
\begin{aligned}
& R_{\text {int }}=0.030 \\
& \theta_{\max }=26.4^{\circ} \\
& h=-8 \rightarrow 8 \\
& k=-8 \rightarrow 8 \\
& l=-17 \rightarrow 17
\end{aligned}
$$

$$
\begin{gathered}
w=1 /\left[\sigma^{2}\left(F_{\mathrm{o}}{ }^{2}\right)+(0.0417 P)^{2}\right. \\
+0.1136 P] \\
\text { where } P=\left(F_{\mathrm{o}}{ }^{2}+2 F_{\mathrm{c}}{ }^{2}\right) / 3
\end{gathered}
$$$$
(\Delta / \sigma)_{\max }=0.001
$$$$
\Delta \rho_{\max }=0.17{\mathrm{e} \AA^{-3}}^{-3}
$$

$\Delta \rho_{\min }=-0.15$ e $\AA^{-3}$

Extinction correction: SHELXL97 Extinction coefficient: 0.046 (11)

Table 1

Hydrogen-bond geometry $\left(\AA{ }^{\circ}\right)$.

\begin{tabular}{lllll}
\hline$D-\mathrm{H} \cdots A$ & $D-\mathrm{H}$ & $\mathrm{H} \cdots A$ & $D \cdots A$ & $D-\mathrm{H} \cdots A$ \\
\hline $\mathrm{N} 3-\mathrm{H} 3 N \cdots \mathrm{N} 1^{\mathrm{i}}$ & $0.957(19)$ & $1.854(19)$ & $2.7978(16)$ & $167.9(16)$ \\
$\mathrm{N} 2-\mathrm{H} 2 N \cdots 3^{\mathrm{ii}}$ & $0.921(18)$ & $1.911(18)$ & $2.8313(18)$ & $175.8(14)$ \\
$\mathrm{N} 2-\mathrm{H} 1 N \cdots \mathrm{O} 1^{\mathrm{iii}}$ & $0.890(18)$ & $1.938(19)$ & $2.8283(16)$ & $177.5(15)$ \\
$\mathrm{O} 3-\mathrm{H} 1 W \cdots \mathrm{O} 2$ & $0.89(2)$ & $1.86(2)$ & $2.7369(15)$ & $172.1(18)$ \\
$\mathrm{O} 3-\mathrm{H} 2 W \cdots \mathrm{O} 1^{\text {iii }}$ & $0.85(2)$ & $1.92(2)$ & $2.7635(15)$ & $173(2)$ \\
\hline
\end{tabular}

Symmetry codes: (i) $x, y, z+1$; (ii) $x+1, y, z$; (iii) $x, y+1, z$.

All $\mathrm{H}$-atom parameters were refined freely; $\mathrm{C}-\mathrm{H}$ distances are in the range 0.953 (16)-0.993 (16) ̊.

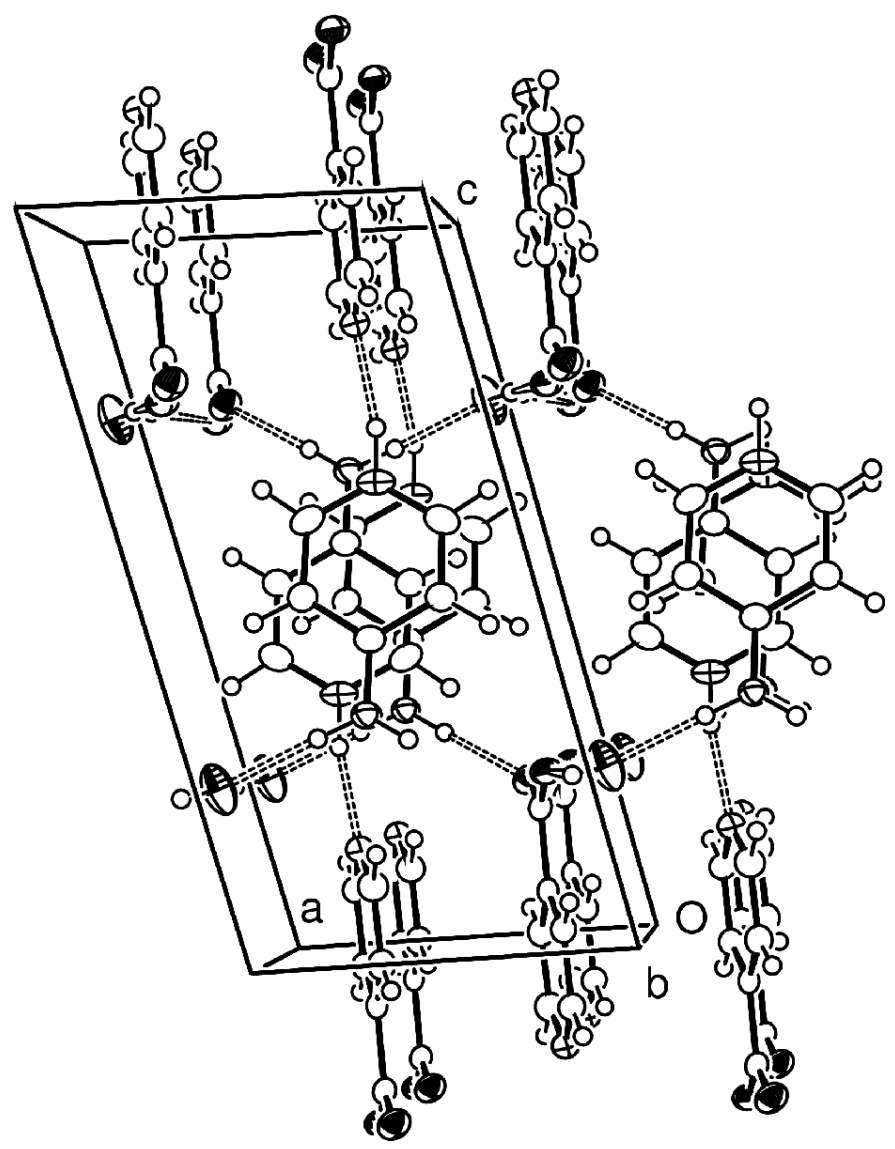

Figure 2

Packing diagram of (I), viewed down the $b$ axis. Dashed lines indicate hydrogen bonds.

Data collection: DENZO (Otwinowski \& Minor, 1997) and COLLECT (Hooft, 1988); cell refinement: DENZO and COLLECT; data reduction: $D E N Z O$; program(s) used to solve structure: $S I R 92$ (Altomare et al., 1994); program(s) used to refine structure: SHELXL97 (Sheldrick, 1997); molecular graphics: ORTEPII (Johnson, 1976); software used to prepare material for publication: SHELXL97.

\section{References}

Altomare, A., Cascarano, G., Giacovazzo, C., Guagliardi, A., Burla, M. C., Polidori, G. \& Camalli, M. (1994). J. Appl. Cryst. 27, 435.

Cowan, J. A., Howard, J. A. K., Leech, M. A. \& Williams, I. D. (2001). Acta Cryst. E57, o563-0565.

Hooft, R. (1988). COLLECT. Nonius BV, Delft, The Netherlands.

Johnson, C. K. (1976). ORTEPII. Report ORNL-5138. Oak Ridge National Laboratory, Tennessee, USA.

Otwinowski, Z. \& Minor, W. (1997). Methods in Enzymology, Vol. 276, Macromolecular Crystallography, Part A, edited by C. W. Carter Jr \& R. M. Sweet, pp. 307-326. New York: Academic Press.

Sheldrick, G. M. (1997). SHELXL97. University of Göttingen, Germany.

Teulon, P., Delaplane, R. G., Olovsson, I. \& Roziere, J. (1985). Acta Cryst. C41, 479-483. 


\section{supporting information}

Acta Cryst. (2005). E61, o333-0334 [https://doi.org/10.1107/S1600536805000887]

\section{4-Aminopyridinium isonicotinate monohydrate}

\section{Alan R. Kennedy and Madeleine Kittner}

4-Aminopyridinium isonicotinate monohydrate

Crystal data

$\mathrm{C}_{5} \mathrm{H}_{7} \mathrm{~N}_{2}{ }^{+} \cdot \mathrm{C}_{6} \mathrm{H}_{4} \mathrm{NO}_{2} \cdot \cdot \mathrm{H}_{2} \mathrm{O}$

$M_{r}=235.24$

Triclinic, $P \overline{1}$

$a=6.7128(3) \AA$

$b=6.7911(2) \AA$

$c=13.6379(8) \AA$

$\alpha=75.830(3)^{\circ}$

$\beta=75.999(2)^{\circ}$

$\gamma=78.326(3)^{\circ}$

$V=578.08(5) \AA^{3}$

Data collection

Nonius KappaCCD

diffractometer

Radiation source: fine-focus sealed tube

Graphite monochromator

$\omega$ and $\varphi$ scans

9253 measured reflections

2348 independent reflections

Refinement

Refinement on $F^{2}$

Least-squares matrix: full

$R\left[F^{2}>2 \sigma\left(F^{2}\right)\right]=0.036$

$w R\left(F^{2}\right)=0.090$

$S=1.04$

2348 reflections

207 parameters

0 restraints

Primary atom site location: structure-invariant direct methods

Secondary atom site location: difference Fourier map
$Z=2$

$F(000)=248$

$D_{\mathrm{x}}=1.351 \mathrm{Mg} \mathrm{m}^{-3}$

Mo $K \alpha$ radiation, $\lambda=0.71073 \AA$

Cell parameters from 2294 reflections

$\theta=1.0-26.4^{\circ}$

$\mu=0.10 \mathrm{~mm}^{-1}$

$T=123 \mathrm{~K}$

Cut plate, colourless

$0.45 \times 0.37 \times 0.10 \mathrm{~mm}$

1848 reflections with $I>2 \sigma(I)$

$R_{\text {int }}=0.030$

$\theta_{\max }=26.4^{\circ}, \theta_{\min }=1.6^{\circ}$

$h=-8 \rightarrow 8$

$k=-8 \rightarrow 8$

$l=-17 \rightarrow 17$

Hydrogen site location: inferred from neighbouring sites

All $\mathrm{H}$-atom parameters refined

$w=1 /\left[\sigma^{2}\left(F_{\mathrm{o}}^{2}\right)+(0.0417 P)^{2}+0.1136 P\right]$

where $P=\left(F_{\mathrm{o}}{ }^{2}+2 F_{\mathrm{c}}{ }^{2}\right) / 3$

$(\Delta / \sigma)_{\max }=0.001$

$\Delta \rho_{\max }=0.17 \mathrm{e} \AA^{-3}$

$\Delta \rho_{\min }=-0.15$ e $\AA^{-3}$

Extinction correction: SHELXL97, $\mathrm{Fc}^{*}=\mathrm{kFc}\left[1+0.001 \mathrm{xFc}^{2} \lambda^{3} / \sin (2 \theta)\right]^{-1 / 4}$

Extinction coefficient: 0.046 (11)

\section{Special details}

Geometry. All e.s.d.'s (except the e.s.d. in the dihedral angle between two 1.s. planes) are estimated using the full covariance matrix. The cell e.s.d.'s are taken into account individually in the estimation of e.s.d.'s in distances, angles and torsion angles; correlations between e.s.d.'s in cell parameters are only used when they are defined by crystal symmetry. An approximate (isotropic) treatment of cell e.s.d.'s is used for estimating e.s.d.'s involving l.s. planes. 
Refinement. Refinement of $F^{2}$ against ALL reflections. The weighted $R$-factor $w R$ and goodness of fit $S$ are based on $F^{2}$, conventional $R$-factors $R$ are based on $F$, with $F$ set to zero for negative $F^{2}$. The threshold expression of $F^{2}>\sigma\left(F^{2}\right)$ is used only for calculating $R$-factors(gt) etc. and is not relevant to the choice of reflections for refinement. $R$-factors based on $F^{2}$ are statistically about twice as large as those based on $F$, and $R$ - factors based on ALL data will be even larger.

Fractional atomic coordinates and isotropic or equivalent isotropic displacement parameters $\left(\hat{A}^{2}\right)$

\begin{tabular}{lllll}
\hline & $x$ & $y$ & $z$ & $U_{\text {iso }} / U_{\text {eq }}$ \\
\hline O1 & $0.22409(15)$ & $0.15150(13)$ & $0.22438(7)$ & $0.0316(3)$ \\
O2 & $0.14675(14)$ & $0.48046(14)$ & $0.23744(7)$ & $0.0290(3)$ \\
O3 & $-0.04150(18)$ & $0.87519(19)$ & $0.24467(10)$ & $0.0483(3)$ \\
N1 & $0.30325(18)$ & $0.5187(2)$ & $-0.14614(9)$ & $0.0343(3)$ \\
N2 & $0.5281(2)$ & $0.90081(17)$ & $0.33110(9)$ & $0.0261(3)$ \\
N3 & $0.35573(19)$ & $0.65430(17)$ & $0.63958(9)$ & $0.0301(3)$ \\
C1 & $0.20050(19)$ & $0.34213(19)$ & $0.18639(10)$ & $0.0233(3)$ \\
C2 & $0.3330(2)$ & $0.3211(2)$ & $-0.09781(11)$ & $0.0345(4)$ \\
C3 & $0.3025(2)$ & $0.2581(2)$ & $0.00823(11)$ & $0.0281(3)$ \\
C4 & $0.23968(18)$ & $0.40528(19)$ & $0.06936(10)$ & $0.0221(3)$ \\
C5 & $0.2115(2)$ & $0.6099(2)$ & $0.01998(10)$ & $0.0251(3)$ \\
C6 & $0.2442(2)$ & $0.6593(2)$ & $-0.08700(11)$ & $0.0303(3)$ \\
C7 & $0.5595(2)$ & $0.64381(19)$ & $0.59471(11)$ & $0.0280(3)$ \\
C8 & $0.6208(2)$ & $0.72207(18)$ & $0.49221(10)$ & $0.0242(3)$ \\
C9 & $0.4716(2)$ & $0.81926(17)$ & $0.43125(10)$ & $0.0220(3)$ \\
C10 & $0.2598(2)$ & $0.82558(19)$ & $0.48123(10)$ & $0.0254(3)$ \\
C11 & $0.2077(2)$ & $0.7433(2)$ & $0.58388(11)$ & $0.0295(3)$ \\
H3N & $0.320(3)$ & $0.604(3)$ & $0.7125(15)$ & $0.051(5)^{*}$ \\
H2 & $0.378(2)$ & $0.219(2)$ & $-0.1425(12)$ & $0.040(4)^{*}$ \\
H1W & $0.030(3)$ & $0.752(3)$ & $0.2392(15)$ & $0.060(6)^{*}$ \\
H2N & $0.668(3)$ & $0.898(2)$ & $0.3041(12)$ & $0.037(4)^{*}$ \\
H3 & $0.325(2)$ & $0.109(2)$ & $0.0397(12)$ & $0.037(4)^{*}$ \\
H2W & $0.048(3)$ & $0.953(3)$ & $0.2359(16)$ & $0.067(6)^{*}$ \\
H1N & $0.433(3)$ & $0.977(3)$ & $0.2965(13)$ & $0.042(5)^{*}$ \\
H5 & $0.167(2)$ & $0.718(2)$ & $0.0595(11)$ & $0.024(3)^{*}$ \\
H6 & $0.225(2)$ & $0.800(2)$ & $-0.1230(12)$ & $0.037(4)^{*}$ \\
H7 & $0.659(2)$ & $0.578(2)$ & $0.6396(12)$ & $0.032(4)^{*}$ \\
H8 & $0.765(2)$ & $0.712(2)$ & $0.4621(11)$ & $0.027(4)^{*}$ \\
H10 & $0.153(2)$ & $0.889(2)$ & $0.4410(12)$ & $0.032(4)^{*}$ \\
H11 & $0.067(2)$ & $0.744(2)$ & $0.6204(12)$ & $0.034(4)^{*}$ \\
& & & &
\end{tabular}

Atomic displacement parameters $\left(\AA^{2}\right)$

\begin{tabular}{lllllll}
\hline & $U^{11}$ & $U^{22}$ & $U^{33}$ & $U^{12}$ & $U^{13}$ & $U^{23}$ \\
\hline O1 & $0.0368(6)$ & $0.0263(5)$ & $0.0301(6)$ & $-0.0058(4)$ & $-0.0126(4)$ & $0.0035(4)$ \\
O2 & $0.0330(5)$ & $0.0318(5)$ & $0.0212(5)$ & $-0.0023(4)$ & $-0.0058(4)$ & $-0.0055(4)$ \\
O3 & $0.0302(6)$ & $0.0324(6)$ & $0.0791(9)$ & $-0.0057(5)$ & $0.0027(6)$ & $-0.0182(6)$ \\
N1 & $0.0254(6)$ & $0.0570(8)$ & $0.0208(6)$ & $-0.0095(5)$ & $-0.0047(5)$ & $-0.0060(5)$ \\
N2 & $0.0269(7)$ & $0.0265(6)$ & $0.0216(6)$ & $-0.0016(5)$ & $-0.0040(5)$ & $-0.0014(5)$ \\
N3 & $0.0437(7)$ & $0.0282(6)$ & $0.0175(6)$ & $-0.0076(5)$ & $-0.0033(5)$ & $-0.0040(5)$
\end{tabular}




\begin{tabular}{lllllll} 
C1 & $0.0192(6)$ & $0.0276(7)$ & $0.0227(7)$ & $-0.0048(5)$ & $-0.0067(5)$ & $-0.0010(5)$ \\
C2 & $0.0286(8)$ & $0.0504(9)$ & $0.0271(8)$ & $-0.0049(7)$ & $-0.0039(6)$ & $-0.0155(7)$ \\
C3 & $0.0237(7)$ & $0.0334(8)$ & $0.0281(8)$ & $-0.0029(6)$ & $-0.0059(6)$ & $-0.0087(6)$ \\
C4 & $0.0152(6)$ & $0.0295(7)$ & $0.0220(7)$ & $-0.0045(5)$ & $-0.0051(5)$ & $-0.0036(5)$ \\
C5 & $0.0208(7)$ & $0.0296(7)$ & $0.0244(7)$ & $-0.0055(5)$ & $-0.0053(5)$ & $-0.0027(6)$ \\
C6 & $0.0249(7)$ & $0.0397(8)$ & $0.0238(7)$ & $-0.0092(6)$ & $-0.0067(6)$ & $0.0035(6)$ \\
C7 & $0.0391(8)$ & $0.0228(7)$ & $0.0254(7)$ & $-0.0045(6)$ & $-0.0132(6)$ & $-0.0048(5)$ \\
C8 & $0.0273(7)$ & $0.0211(6)$ & $0.0253(7)$ & $-0.0036(5)$ & $-0.0060(6)$ & $-0.0064(5)$ \\
C9 & $0.0294(7)$ & $0.0157(6)$ & $0.0215(7)$ & $-0.0034(5)$ & $-0.0040(5)$ & $-0.0058(5)$ \\
C10 & $0.0280(7)$ & $0.0220(6)$ & $0.0244(7)$ & $-0.0013(5)$ & $-0.0045(6)$ & $-0.0044(5)$ \\
C11 & $0.0336(8)$ & $0.0264(7)$ & $0.0261(8)$ & $-0.0049(6)$ & $0.0014(6)$ & $-0.0081(5)$ \\
\hline
\end{tabular}

Geometric parameters $\left(\AA,{ }^{\circ}\right)$

\begin{tabular}{|c|c|c|c|}
\hline $\mathrm{O} 1-\mathrm{C} 1$ & $1.2640(15)$ & $\mathrm{C} 3-\mathrm{C} 4$ & $1.3924(18)$ \\
\hline $\mathrm{O} 2-\mathrm{C} 1$ & $1.2486(15)$ & $\mathrm{C} 3-\mathrm{H} 3$ & $0.993(16)$ \\
\hline $\mathrm{O} 3-\mathrm{H} 1 \mathrm{~W}$ & $0.89(2)$ & $\mathrm{C} 4-\mathrm{C} 5$ & $1.3844(18)$ \\
\hline $\mathrm{O} 3-\mathrm{H} 2 \mathrm{~W}$ & $0.85(2)$ & $\mathrm{C} 5-\mathrm{C} 6$ & $1.3873(19)$ \\
\hline $\mathrm{N} 1-\mathrm{C} 6$ & $1.3370(19)$ & $\mathrm{C} 5-\mathrm{H} 5$ & $0.971(14)$ \\
\hline $\mathrm{N} 1-\mathrm{C} 2$ & $1.341(2)$ & $\mathrm{C} 6-\mathrm{H} 6$ & $0.961(16)$ \\
\hline $\mathrm{N} 2-\mathrm{C} 9$ & $1.3342(16)$ & $\mathrm{C} 7-\mathrm{C} 8$ & $1.3610(19)$ \\
\hline $\mathrm{N} 2-\mathrm{H} 2 \mathrm{~N}$ & $0.921(18)$ & $\mathrm{C} 7-\mathrm{H} 7$ & $0.981(16)$ \\
\hline $\mathrm{N} 2-\mathrm{H} 1 \mathrm{~N}$ & $0.890(18)$ & $\mathrm{C} 8-\mathrm{C} 9$ & $1.4129(19)$ \\
\hline N3-C11 & $1.3516(19)$ & $\mathrm{C} 8-\mathrm{H} 8$ & $0.954(15)$ \\
\hline $\mathrm{N} 3-\mathrm{C} 7$ & $1.3520(19)$ & $\mathrm{C} 9-\mathrm{C} 10$ & $1.4171(19)$ \\
\hline $\mathrm{N} 3-\mathrm{H} 3 \mathrm{~N}$ & $0.957(19)$ & $\mathrm{C} 10-\mathrm{C} 11$ & $1.3614(19)$ \\
\hline $\mathrm{C} 1-\mathrm{C} 4$ & $1.5192(18)$ & $\mathrm{C} 10-\mathrm{H} 10$ & $0.975(16)$ \\
\hline $\mathrm{C} 2-\mathrm{C} 3$ & $1.380(2)$ & $\mathrm{C} 11-\mathrm{H} 11$ & $0.953(16)$ \\
\hline $\mathrm{C} 2-\mathrm{H} 2$ & $0.987(16)$ & & \\
\hline $\mathrm{H} 1 \mathrm{~W}-\mathrm{O} 3-\mathrm{H} 2 \mathrm{~W}$ & $106.0(19)$ & $\mathrm{C} 4-\mathrm{C} 5-\mathrm{H} 5$ & $120.6(8)$ \\
\hline $\mathrm{C} 6-\mathrm{N} 1-\mathrm{C} 2$ & $117.13(12)$ & $\mathrm{C} 6-\mathrm{C} 5-\mathrm{H} 5$ & $120.2(8)$ \\
\hline $\mathrm{C} 9-\mathrm{N} 2-\mathrm{H} 2 \mathrm{~N}$ & $117.4(10)$ & $\mathrm{N} 1-\mathrm{C} 6-\mathrm{C} 5$ & $123.35(13)$ \\
\hline $\mathrm{C} 9-\mathrm{N} 2-\mathrm{H} 1 \mathrm{~N}$ & $119.6(11)$ & $\mathrm{N} 1-\mathrm{C} 6-\mathrm{H} 6$ & $116.0(9)$ \\
\hline $\mathrm{H} 2 \mathrm{~N}-\mathrm{N} 2-\mathrm{H} 1 \mathrm{~N}$ & $121.7(15)$ & $\mathrm{C} 5-\mathrm{C} 6-\mathrm{H} 6$ & $120.7(9)$ \\
\hline $\mathrm{C} 11-\mathrm{N} 3-\mathrm{C} 7$ & $120.86(12)$ & $\mathrm{N} 3-\mathrm{C} 7-\mathrm{C} 8$ & $120.80(13)$ \\
\hline $\mathrm{C} 11-\mathrm{N} 3-\mathrm{H} 3 \mathrm{~N}$ & $121.0(11)$ & $\mathrm{N} 3-\mathrm{C} 7-\mathrm{H} 7$ & $116.8(9)$ \\
\hline $\mathrm{C} 7-\mathrm{N} 3-\mathrm{H} 3 \mathrm{~N}$ & $118.1(11)$ & $\mathrm{C} 8-\mathrm{C} 7-\mathrm{H} 7$ & $122.4(9)$ \\
\hline $\mathrm{O} 2-\mathrm{C} 1-\mathrm{O} 1$ & $125.12(12)$ & $\mathrm{C} 7-\mathrm{C} 8-\mathrm{C} 9$ & $120.31(13)$ \\
\hline $\mathrm{O} 2-\mathrm{C} 1-\mathrm{C} 4$ & $118.15(11)$ & $\mathrm{C} 7-\mathrm{C} 8-\mathrm{H} 8$ & $119.3(9)$ \\
\hline $\mathrm{O} 1-\mathrm{C} 1-\mathrm{C} 4$ & $116.73(11)$ & $\mathrm{C} 9-\mathrm{C} 8-\mathrm{H} 8$ & $120.4(9)$ \\
\hline $\mathrm{N} 1-\mathrm{C} 2-\mathrm{C} 3$ & $123.37(13)$ & $\mathrm{N} 2-\mathrm{C} 9-\mathrm{C} 8$ & $121.35(12)$ \\
\hline $\mathrm{N} 1-\mathrm{C} 2-\mathrm{H} 2$ & $116.2(9)$ & $\mathrm{N} 2-\mathrm{C} 9-\mathrm{C} 10$ & $121.65(12)$ \\
\hline $\mathrm{C} 3-\mathrm{C} 2-\mathrm{H} 2$ & $120.4(9)$ & $\mathrm{C} 8-\mathrm{C} 9-\mathrm{C} 10$ & $117.00(12)$ \\
\hline $\mathrm{C} 2-\mathrm{C} 3-\mathrm{C} 4$ & $119.18(13)$ & $\mathrm{C} 11-\mathrm{C} 10-\mathrm{C} 9$ & $120.09(13)$ \\
\hline $\mathrm{C} 2-\mathrm{C} 3-\mathrm{H} 3$ & $119.7(9)$ & $\mathrm{C} 11-\mathrm{C} 10-\mathrm{H} 10$ & $120.8(9)$ \\
\hline $\mathrm{C} 4-\mathrm{C} 3-\mathrm{H} 3$ & $121.1(9)$ & $\mathrm{C} 9-\mathrm{C} 10-\mathrm{H} 10$ & $119.1(9)$ \\
\hline $\mathrm{C} 5-\mathrm{C} 4-\mathrm{C} 3$ & $117.80(12)$ & $\mathrm{N} 3-\mathrm{C} 11-\mathrm{C} 10$ & $120.93(13)$ \\
\hline
\end{tabular}




\begin{tabular}{llll}
$\mathrm{C} 5-\mathrm{C} 4-\mathrm{C} 1$ & $121.38(12)$ & $\mathrm{N} 3-\mathrm{C} 11-\mathrm{H} 11$ & $116.3(9)$ \\
$\mathrm{C} 3-\mathrm{C} 4-\mathrm{C} 1$ & $120.81(11)$ & $\mathrm{C} 10-\mathrm{C} 11-\mathrm{H} 11$ & $122.8(9)$ \\
$\mathrm{C} 4-\mathrm{C} 5-\mathrm{C} 6$ & $119.16(13)$ & & \\
& & & \\
$\mathrm{C} 6-\mathrm{N} 1-\mathrm{C} 2-\mathrm{C} 3$ & $1.1(2)$ & $\mathrm{C} 2-\mathrm{N} 1-\mathrm{C} 6-\mathrm{C} 5$ & $-0.5(2)$ \\
$\mathrm{N} 1-\mathrm{C} 2-\mathrm{C} 3-\mathrm{C} 4$ & $-0.7(2)$ & $\mathrm{C} 4-\mathrm{C} 5-\mathrm{C} 6-\mathrm{N} 1$ & $-0.23(1)$ \\
$\mathrm{C} 2-\mathrm{C} 3-\mathrm{C} 4-\mathrm{C} 5$ & $-0.27(19)$ & $\mathrm{C} 11-\mathrm{N} 3-\mathrm{C} 7-\mathrm{C} 8$ & $1.17(18)$ \\
$\mathrm{C} 2-\mathrm{C} 3-\mathrm{C} 4-\mathrm{C} 1$ & $178.83(12)$ & $\mathrm{N} 3-\mathrm{C} 7-\mathrm{C} 8-\mathrm{C} 9$ & $178.61(12)$ \\
$\mathrm{O} 2-\mathrm{C} 1-\mathrm{C} 4-\mathrm{C} 5$ & $-1.76(18)$ & $\mathrm{C} 7-\mathrm{C} 8-\mathrm{C} 9-\mathrm{N} 2$ & $-1.56(17)$ \\
$\mathrm{O} 1-\mathrm{C} 1-\mathrm{C} 4-\mathrm{C} 5$ & $177.80(11)$ & $\mathrm{C} 7-\mathrm{C} 8-\mathrm{C} 9-\mathrm{C} 10$ & $-179.09(12)$ \\
$\mathrm{O} 2-\mathrm{C} 1-\mathrm{C} 4-\mathrm{C} 3$ & $179.18(11)$ & $\mathrm{N} 2-\mathrm{C} 9-\mathrm{C} 10-\mathrm{C} 11$ & $1.08(18)$ \\
$\mathrm{O} 1-\mathrm{C} 1-\mathrm{C} 4-\mathrm{C} 3$ & $-1.26(17)$ & $\mathrm{C} 8-\mathrm{C} 9-\mathrm{C} 10-\mathrm{C} 11$ & $-0.26(19)$ \\
$\mathrm{C} 3-\mathrm{C} 4-\mathrm{C} 5-\mathrm{C} 6$ & $0.74(18)$ & $\mathrm{C} 7-\mathrm{N} 3-\mathrm{C} 11-\mathrm{C} 10$ & $-0.19(19)$ \\
$\mathrm{C} 1-\mathrm{C} 4-\mathrm{C} 5-\mathrm{C} 6$ & $-178.35(11)$ & $\mathrm{C} 9-\mathrm{C} 10-\mathrm{C} 11-\mathrm{N} 3$ & \\
\hline
\end{tabular}

Hydrogen-bond geometry $\left(\AA,{ }^{\circ}\right)$

\begin{tabular}{lllll}
\hline$D-\mathrm{H} \cdots A$ & $D-\mathrm{H}$ & $\mathrm{H} \cdots A$ & $D \cdots A$ & $D-\mathrm{H} \cdots A$ \\
\hline $\mathrm{N} 3-\mathrm{H} 3 N \cdots \mathrm{N} 1^{\mathrm{i}}$ & $0.957(19)$ & $1.854(19)$ & $2.7978(16)$ & $167.9(16)$ \\
$\mathrm{N} 2-\mathrm{H} 2 N \cdots \mathrm{O} 3^{\text {ii }}$ & $0.921(18)$ & $1.911(18)$ & $2.8313(18)$ & $175.8(14)$ \\
$\mathrm{N} 2-\mathrm{H} 1 N^{\cdots} \mathrm{O} 1^{\text {iii }}$ & $0.890(18)$ & $1.938(19)$ & $2.8283(16)$ & $177.5(15)$ \\
$\mathrm{O} 3-\mathrm{H} 1 W^{\cdots} \cdots 2$ & $0.89(2)$ & $1.86(2)$ & $2.7369(15)$ & $172.1(18)$ \\
$\mathrm{O} 3-\mathrm{H} 2 W \cdots \mathrm{O} 1^{\text {iii }}$ & $0.85(2)$ & $1.92(2)$ & $2.7635(15)$ & $173(2)$
\end{tabular}

Symmetry codes: (i) $x, y, z+1$; (ii) $x+1, y, z$; (iii) $x, y+1, z$. 